\title{
Value of Dual Reports on Routine Electrocardiograms
}

\author{
J. SEYMOUR AND N. CONWAY \\ From the Department of Cardiology, Middlesex Hospital, London W.1
}

The results of many investigations are liable to be reported differently by different observers. This is due to a combination of doubt, error, and inconsistency which has been termed "observer variation" (Davies, 1958). Discrepancies attributable to this cause have been shown, for example, in reports on chest $x$-rays (Whyte, 1955), in the evaluation of bronchoscopic findings (Houghton and Joules, 1953), and in the counting of red cells (Biggs and Macmillan, 1948), but the reliability of reports on routine electrocardiograms is a subject to which little attention has been paid. This is probably because there is often doubt about the correct interpretation of many electrocardiograms. Davies (1958), for instance, showed 100 selected electrocardiograms to 9 experienced observers, yet obtained unanimous agreement about the diagnosis in only 29 tracings. In 49 more he was obliged to accept a majority view, and 22 were so disputed that he removed them entirely from his analysis. However, his investigation was artificial in several respects. The electrocardiograms were specially selected and included 50 thought to show myocardial infarction-a notorious area of dispute. Again, only three reports were allowed, namely "normal", "abnormal", or "infarction", and all the tracings were reported at a single sitting - a tiring task. It is likely that these features greatly contributed to the startling divergencies of opinion he obtained, and the relevance of such a study to the evaluation of routine reports on electrocardiograms is open to question.

We have analysed reports on the electrocardiograms recorded over a 2-month period in a busy general hospital. Each record was reported independently by both of us in order to discover the incidence of agreement. We have accepted such agreement as indicating the issue of a "correct" report. There are fallacies in this approach, but it has the virtue of practicality in that duplicate

Received March 13, 1969. reporting, though costly in time, is not outside the capacity of any hospital, and it would be helpful to know if this simple step would decrease observer variation.

\section{SUBJECTS AND Methods}

In this hospital all electrocardiograms, except those recorded on patients under the care of the Cardiac Department, are routinely reported by one or other of us. Over the period of the study each reported tracing was retained, after issue of the usual photocopy, for separate reporting by the observer who had not yet seen it. The original request slip was kept, and with the first report concealed, a second one was prepared. This was the "first reading". No attempt was made to alter the customary circumstances of the daily reporting sessions, either in respect of timing or of special scrutiny. In all cases in which the two reports differed the electrocardiogram was later re-examined by both of us together-the "second reading". Records in which agreement had been reached on the first reading were not examined further. All cardiograms were recorded on a Devices direct writing machine (Model C.I.M.).

\section{ResULTS}

The total number of electrocardiograms read was 1222. Agreement was reached at the first reading in 1073, and at the second reading in 114 more, leaving 35 tracings (3\% of the total) in which the report could not be agreed. Thus it was possible to agree ultimately on a report for 1187 electrocardiograms ( $97 \%$ of the total).

Table I analyses the performance of each observer reporting the electrocardiograms by himself in the light of the report which was subsequently agreed to be "correct". Observer " $A$ " issued 1133 "correct" reports (92\% of the total) and observer " $B$ " issued 1127 "correct" reports (92\% of the total). Observer " $A$ " admitted error in 54 reports ( $5 \%$ of the total) and observer " $B$ " in 60 reports (5\% of the total). These errors are analysed in detail below, and the data are summarized (see Tables IV and V). 
TABLE I

ANALYSIS OF EACH OBSERVER'S REPORTS

\begin{tabular}{l|c|c}
\hline & \multicolumn{2}{|c}{ Observer } \\
\cline { 2 - 3 } & A & B \\
\hline Correct reports issued & 1133 & 1127 \\
Incorrect reports amended after discussion & 35 & 25 \\
(a) Abnormality missed & 25 \\
(b) Electrocardiogram misinterpreted & 19 & 35 \\
\hline Total reports agreed & 1187 & 1187 \\
Reports finally disputed & 35 & 35 \\
\hline Grand total & 1222 & 1222 \\
\hline
\end{tabular}

Table II analyses the reports issued on the 1073 tracings in which agreement had occurred on the first reading. In this analysis, as in the later ones in this paper, only the more important diagnosis has been given for reasons of clarity, e.g. myocardial infarction and atrial fibrillation have been listed as myocardial infarction. It follows that the apparent incidence of such abnormalities as ectopic beats, digitalis effect, and $P$ mitrale, is unusually low. Recent and old myocardial infarctions were not separately analysed.

TABLE II

ANALYSIS OF ELECTROCARDIOGRAM REPORTS AGREED AT FIRST READING BY BOTH OBSERVERS

\begin{tabular}{l|r}
\hline Normal & 556 \\
Consistent with myocardial ischaemia & 191 \\
Myocardial infarction & 82 \\
Left ventricular hypertrophy & 68 \\
Right ventricular hypertrophy & 6 \\
Left bundle-branch block & 14 \\
Right bundle-branch block & 16 \\
Atrial fibrillation & 39 \\
Ectopic beats & 30 \\
Heart block & 8 \\
Other arrhythmias & 18 \\
Miscellaneous & 45 \\
\hline Total & 1073 \\
\hline
\end{tabular}

Table III analyses the 35 electrocardiograms in which dispute could not be resolved. The major difficulty in this group lay in deciding the significance of slight ST and T abnormalities, and in the evaluation of isolated $Q$ waves.

TABLE III

ANALYSIS OF CAUSES OF FINAL DISAGREEMENT IN 35 ELECTROCARDIOGRAMS

ST-T wave abnormalities versus normality Presence of infarction in ischaemic tracing Infarction versus normality

Ischaemia in presence of left ventricular hypertrophy

Axis dispute

Presence of right bundle-branch block Rhythm dispute

Total

$\begin{array}{r}17 \\ 8 \\ 3 \\ 1 \\ 2 \\ 2 \\ 2 \\ \hline 35 \\ \hline\end{array}$

On 114 occasions the initial cardiogram reports differed. When these tracings were re-examined observer " $A$ " was considered to have erred 54 times: 35 of these errors were due to missing a definite abnormality and 19 were due to reporting abnormalities which were not, in fact, present. Observer " $B$ " was considered to have erred on 60 occasions: 25 of these errors were due to missing a definite abnormality, and 35 were due to reporting abnormalities which were not present (see Table I).

The diagnoses missed by each observer are listed in Table IV. Again, the interpretation of minor ST and $T$ abnormalities posed the main difficulty. Myocardial infarction was missed in 9 and 5 instances, respectively. In part these misreports arose from the problems in terminology when electrocardiograms showed localized, symmetrical $T$ wave inversions without $Q$ waves, the presence of ischaemia not being in doubt.

TABLE IV

DIAGNOSES MISSED BY EACH OBSERVER

\begin{tabular}{l|c|c|c}
\hline & \multicolumn{2}{|c|}{ Observer } & \\
\cline { 2 - 3 } & A & B & Total \\
\hline Ischaemia & 11 & 11 & 22 \\
Myocardial infarction & 9 & 5 & 14 \\
Left ventricular hypertrophy & 8 & 4 & 12 \\
Prolonged P-R interval & 3 & 1 & 4 \\
Left axis deviation & 1 & 0 & 1 \\
Right axis deviation & 1 & 0 & 1 \\
Atrial fibrillation & 0 & 2 & 2 \\
Other & 2 & 2 & 4 \\
\hline Total & 35 & 25 & 60 \\
\hline
\end{tabular}

TABLE V

ABNORMALITIES REPORTED BY EACH OBSERVER WHICH WERE NOT CONFIRMED AT SECOND READING

\begin{tabular}{l|r|r|r}
\hline & \multicolumn{2}{|c|}{ Observer } & \multirow{2}{*}{ Total } \\
\cline { 2 - 3 } & A & B & Tot \\
\hline Ischaemia & 10 & 19 & 29 \\
Myocardial infarction & 1 & 3 & 4 \\
Left ventricular hypertrophy & 5 & 5 & 10 \\
Ischaemia in presence of left ventricu- & 1 & 6 & 7 \\
lar hypertrophy & 1 & 2 & 4 \\
Miscellaneous & 2 & 35 & 54 \\
\hline Total & 19 & & \\
\hline
\end{tabular}

Table $\mathrm{V}$ analyses the incorrect diagnoses made by each observer. Once again, the majority of these errors arose from misinterpretation of minor ST and $T$ abnormalities, though on 4 occasions myocardial infarction was reported incorrectly.

\section{Discussion}

The electrocardiogram is often of considerable help in the evaluation of heart disease, but it is 
important to realize its limitations. Some electrocardiographic abnormalities are diagnostic, but other patterns may be produced by a variety of pathological processes. Furthermore, the borderline between normality and abnormality is ill defined, and a normal tracing may coexist with frank cardiac pathology. For these reasons it follows that the cardiogram is of value only if considered critically in the clinical context, and a report on it must be regarded as a guide and no more. This study examines whether the quality of such guidance can be easily improved.

The results show that conflicting reports were initially recorded in 149 instances. In 114 of these tracings one or other observer, when shown his original report, realized he had been at fault, and was prepared to amend it. Each observer was more or less equally to blame. " $A$ " missed more abnormality than " $B$ ", though he misinterpreted fewer normal tracings. The interpretation of 35 electrocardiograms $(3 \%)$ remained in dispute. In the remaining 1073 electrocardiograms the initial reports agreed so that finally agreement was reached in $1193(97 \%)$.

The major cause of disagreement and error was the assessment of minor ST-T abnormalities suggestive of ischaemia. Half the tracings in which dispute could not be reconciled, a similar proportion of incorrect diagnoses, and a third of all diagnoses missed fall in this group. This is not surprising since this segment of the cardiogram is sensitive to many factors (not all of which are known to the reporter), and the limits of normality are poorly defined. The next diagnosis causing difference of opinion was myocardial infarction. The presence of acute infarction was rarely a problem, but the diagnosis of old infarction sometimes caused difficulty. The significance of $Q$ waves in isolated posterior leads and the interpretation of symmetrically inverted $\mathrm{T}$ waves accounted for most disputes. For these reasons the diagnosis of old infarction was missed on a total of 14 occasions. The last diagnosis to cause difficulty was left ventricular hypertrophy, which was missed 12 times: on 5 occasions it was incorrectly reported by each of us. Mostly these tracings showed borderline changes; on a few occasions it was not realized that the tracing had been recorded at half standardization.

Thus, by duplicate reporting and subsequent discussion approximately 5 per cent of the electrocardiogram reports by each observer were corrected (" $A$ " 54, and " $B$ " 60). The benefits were equally distributed between the detection of missed diagnoses and the amendment of incorrect ones. This result must be treated with caution, however, since nearly half the corrections were applied to tracings that showed minor ST and T abnormalities, the assessment of which is often a matter of opinion rather than fact. In 3 per cent of electrocardiograms combined discussion was not helpful.

The explanation for misreports later seen to be obviously incorrect is, for the most part, obscure. In some instances the probable answer was inadequate appreciation of details on the request slip, such as age or digitalis administration. In others it may have been bias produced by a positive clinical assessment (e.g. "typical angina" or "acute infarction yesterday"). Lastly there are the imponderable factors of fatigue or distraction while reporting. These aspects of observer variation are difficult to evaluate in retrospect, and their importance cannot be easily examined.

This study suggests that in the course of day-today reporting of routine electrocardiograms at least 5 per cent of tracings will be found to be incorrectly interpreted by a second observer. The percentage no doubt, would rise if yet more observers were enlisted, but in that case the practical character of the exercise would disappear. Formal dual reporting of every electrocardiogram and combined consideration of disputed tracings is therefore likely to yield a small dividend in the form of more reliable guidance to the clinician. The benefit must be weighed against the problems of organization, staffing, and delay in the issue of reports which this measure entails.

\section{SUMMARY}

During a 2-month period 1222 routine electrocardiograms were reported independently by two observers. Identical reports were issued in 92 per cent. Each observer was in error in approximately 5 per cent of tracings, thus permitting correction of the initial report. Reports on the remaining 3 per cent of electrocardiograms remained in dispute. The sources of error and disagreement are analysed, and the value of formal dual reporting of electrocardiograms is discussed.

We are grateful to Dr. Walter Somerville for his encouragement and advice. We thank the technicians of the Department of Cardiology for their help during the period of this study.

\section{REFERENCES}

Biggs, R., and Macmillan, R. L. (1948). The error of the red cell count. F. clin. Path., 1, 288.

Davies, L. G. (1958). Observer variation in reports on electrocardiograms. Brit. Heart $\mathcal{F}$., 20, 153.

Houghton, L. E., and Joules, F. E. (1953). Tuberculous tracheobronchitis. Tubercle (Edinb.), 34, 74.

Whyte, H. M. (1955). Differences in opinions of radiological changes in pulmonary tuberculosis. Aust. Ann. Med., 4, 100. 